\title{
Very Early Arterial Ischemic Stroke in Premature Infants
}

\author{
Meredith R. Golomb, MD, MSc ${ }^{1}$, Bhuwan P. Garg, MBBS ${ }^{1}$, Mary Edwards-Brown, MD ${ }^{2}$, and \\ Linda S. Williams, MD $3,4,5$ \\ ${ }^{1}$ Division of Pediatric Neurology, Department of Neurology, Indiana University School of Medicine \\ ${ }^{2}$ Department of Neuroradiology, Indiana University School of Medicine \\ ${ }^{3}$ Roudebush Veterans' Affairs Medical Center Health Services Research and Development Service, \\ Indiana University School of Medicine \\ ${ }^{4}$ Regenstrief Institute, Indiana University School of Medicine \\ ${ }^{5}$ Department of Nuerology, Indiana University School of Medicine
}

\begin{abstract}
There have been few descriptions of early stroke in the premature infant. We present 23 infants born between 23 and 35 weeks gestational ages with focal arterial ischemic stroke occurring before 44 weeks gestational age. Ten (43\%) were male. Five children (22\%) were half of a twin pair; no cotwin died. The most commonly affected territory was the middle cerebral artery territory. Three children with extreme prematurity ( $<=26$ weeks) had cerebellar infarcts. Twelve children had unilateral or bilateral intraventricular hemorrhages, which were grade 3 or higher in 8 . Twelve children had white matter injury (periventricular lukomalacia and/or hypoxic ischemic encephalopathy). Most children had multiple additional comorbidities, and median neonatal intensive care unit stay was 63 days (range 14-365). One child died in the neonatal intensive care unit. All 22 survivors were left with disabilities. Seventeen (77\%) had cerebral palsy, $10(45 \%)$ had epilepsy, and $17(77 \%)$ had cognitive impairment. Arterial ischemic stroke appears to add to the neurological disabilities commonly associated with prematurity.
\end{abstract}

\section{Introduction}

Arterial ischemic stroke can occur in infants born at any gestational age, but there are few descriptions of very early arterial ischemic stroke in premature children. Many outcome studies of perinatal stroke exclude premature children because these children often have additional neurological complications of prematurity such as intraventricular hemorrhage and periventricular leukomalacia, making it difficult to compare them with term children who have isolated, focal cerebral injury[1-3]. Premature infants are often medically fragile in the perinatal period and the months beyond, which limits cerebral imaging and contributes to the dearth of data on this subject. We describe the presentation, clinical course, and outcomes of 23 premature children with stroke before 44 weeks gestational age who were seen at our institution.

(C) 2008 Elsevier Inc. All rights reserved.

Corresponding author: Meredith R. Golomb MD, MSc, Indiana University School of Medicine, Building XE 040,575 West Dr., Indianapolis, IN 46202, Email: mgolomb@iupui.edu, Phone: 317-278-5450, Fax: 317-274-3622.

Publisher's Disclaimer: This is a PDF file of an unedited manuscript that has been accepted for publication. As a service to our customers we are providing this early version of the manuscript. The manuscript will undergo copyediting, typesetting, and review of the resulting proof before it is published in its final citable form. Please note that during the production process errors may be discovered which could affect the content, and all legal disclaimers that apply to the journal pertain. 


\section{Methods}

Children in this study were identified by a combination of neurology clinic record review from September 1989-September 2006, patient referral during this period, and ICD-9 code searches using codes 342, 433-438 and 767 from May 1, 1999 to May 1 2004. Further ICD-9 searches were not performed after the limitations of this searches were discovered.[4]. Children were included if they were born before 36 weeks gestation age and had cranial computed tomography and/or magnetic resonance imaging demonstrating focal arterial ischemic infarct which was known to or appeared to occur before 44 weeks gestational age. Forty-four weeks gestational age was chosen as the cut-off point because this would be the end of the perinatal period if the child had been born at term; for many cases, radiographic confirmation of infarction within the actual perinatal period (up to 28 days after birth) was not possible due to the medical instability of the child. Children with hemispheric atrophy ipsilateral to a grade 3 or 4 intraventricular hemorrhage and no other signs of focal cerebral injury in an arterial territory were not included. Standardized medical record reviews were performed to collect the following data from hospital and clinic notes, radiographic films, and laboratory records: gender and ethnicity; gestational age at birth; gestational and perinatal complications and comorbidities; cranial radiographic findings; neonatal intensive care unit course; and motor, cognitive, and epilepsy outcomes. Radiographic findings were described in terms of infarcted arterial territories, hemorrhagic transformation of infarcted territory, intraventricular hemorrhage, and white matter injury (periventricular leukomalacia or hypoxic-ischemic encephalopathy). The authors re-reviewed radiographic films whenever they could be obtained (18 cases); for the remaining 5 cases, radiographic reports, hospital notes, and clinic notes were used. Motor outcomes were defined as mild motor abnormality (abnormality of tone or reflexes not interfering with function) or cerebral palsy (static abnormality of tone or movement attributable to cerebral injury and interfering with function). Cognitive/speech outcomes were defined as mild impairments (requiring modified classes, or meeting Indiana state requirements for developmental or speech therapy but able to learn and likely to eventually live independently) or moderate to severe (being unlikely to ever live independently). Epilepsy was defined as the presence of clinical or electrographic seizures. Mild epilepsy was defined as less than or equal to one seizure per month while on medication, while moderate-severe epilepsy was defined as more than one seizure per month while on medication. These outcome definitions have been used in previously reported work[5].

\section{Results}

\section{Patient population}

Twenty-three patients were identified: 10 (43\%) were male. Ethnic backgrounds were: Caucasian (16; 70\%), African-American (5;22\%), Hispanic (2; 9\%). None were Asian or of mixed ethnicity. Gestational age at birth ranged from 23-35 weeks: 9 were born at 27 weeks or earlier. Birth weights ranged from 535 to 2786 grams. (Table 1).

\section{Gestational complications}

Five children (22\%) were part of twin gestations; none were part of triplet or higher multiple gestations. One of these children had twin-twin transfusion syndrome. None of the 5 co-twins died; no co-twin had arterial ischemic stroke. None of the children in this study were conceived with the aid of fertility treatments (artificial insemination, medications, or in-vitro fertilization). Perinatal complications in the mother or fetus included: abnormal gestational bleeding in the mother $(5 ; 22 \%)$, gestational diabetes $(1 ; 4 \%)$, preeclampsia/hypertension $(4 ; 17 \%)$, maternal infection $(7 ; 30 \%)$, maternal trauma $(1 ; 4 \%)$, placenta previa $(1 ; 4 \%)$, maternal smoking $(4$; $17 \%)$ maternal illegal drug use (2; $9 \%, 1$ used marijuana and cocaine while 1 used marijuana and alcohol, both women smoked tobacco), maternal prothrombotic disorder with deep venous 
thrombosis $(1 ; 4 \%)$. Maternal age was available for 20 subjects. Median maternal age for these 20 women was 28 years (range 16-38 years); 5 mothers gave birth before 20 years of age and 4 mothers gave birth after 35 years of age.

\section{Delivery issues}

Information on delivery was available for 22 of the 23 patients (96\%). Ten children were born by spontaneous vaginal delivery. One child was delivered by spontaneous vaginal delivery with vacuum assistance. Twelve children were born by cesarean section which was emergent in 6 . Four children had breech presentation; 1 was delivered by spontaneous vaginal delivery and 3 by cesarean section; none of these were emergent. Five children had transverse lie presentation; each was delivered by cesarean section, 3 of which were emergent. Six children had fetal distress; 4 delivered by emergent cesarean section, 1 by vaginal delivery with vacuum assistance and 1 by spontaneous vaginal delivery. Seven mothers were treated with steroids before delivery to promote lung development; 6 received magnesium sulfate for tocolysis. Ten children received surfarctant with intubation; all of these children were 31 weeks' gestational age or less at birth. Twelve children required intubation immediately after delivery, and all but 1 of these children were under 32 weeks' gestational age at birth; 3 of these required highfrequency ventilation shortly after delivery due to poor response to intubation. One child later required extracorporeal membrane oxygenation at 2 months of age due to increasing respiratory failure. Two children required chest compressions at birth; 1 of these required epinephrine.

\section{Clinical Presentations}

All children (100\%) presented in the neonatal period, at a median age of 1 day (range 1-4). The most common presentations during the neonatal period were: respiratory difficulty or apnea $19(83 \%)$, seizures $7(30 \%)$, poor feeding $6(26 \%)$, abnormal tone $5(22 \%)$. Two children developed symptoms of hemiparesis after the neonatal period which prompted further evaluation.

\section{Diagnosis of arterial ischemic stroke and radiographic findings $(n=23)$}

All children had imaging suggesting the occurrence of stroke within the first 44 weeks of gestational age. Children were initially diagnosed by magnetic resonance imaging (5) at a median age of 72 days (range $2-121$ ), computed tomography (10) at a median age of 22.5 days (range $2-72$ ), and cranial ultrasound (2) at a median age of 35 days (range $7-63$ ). The age of first diagnosis was unclear in 6 children, but all children had at least one cranial computed tomography scan or magnetic resonance imaging study confirming focal arterial ischemic infarct likely to have occurred in the first 44 gestational weeks of life. Eighteen children had the diagnosis confirmed by magnetic resonance imaging. The most commonly affected territory was the middle cerebral artery territory. (Figure 1) Ten children had small branch middle cerebral artery infarct ( 8 left, 2 right) 1 had a large-branch left middle cerebral artery infarct, and one had unilateral involvement of the left middle and posterior cerebral artery territories. Two children had small thalamic infarcts, most likely posterior cerebral artery territory (1 left, 1 right). One child had a large right posterior cerebral artery infarct. Five children had bilateral cerebral infarcts: one had bilateral basal ganglia infarcts, one had bilateral posterior cerebral artery infarcts, one had bilateral anterior cerebral artery infarct, one had multiple small infarcts in multiple territories, and one had bilateral posterior watershed infarcts together with a left small-branch middle cerebral artery infarct. Three children had cerebellar infarcts that appeared to involve the posterior inferior cerebellar arteries: one left, one right, one bilateral. (Figure 2) Four children had hemorrhagic infarcts.

Twelve of 23 children had unilateral or bilateral intraventricular hemorrhage, which was grade 3 or higher in 8 . Twelve children had white matter injury (periventricular leukomalacia and/ or hypoxic-ischemic encephalopathy). Seven children had some degree of both intraventricular 
hemorrhage and white matter injury. Two children had limited documentation of cerebral injury beyond their infarct; their original films were not available for re-review. Seven children developed hydrocephalus secondary to intraventricular hemorrhage or meningitis requiring ventriculoperitoneal shunt placement.

\section{Neonatal intensive care unit course and neurological findings while in the unit}

Median length of neonatal intensive care unit stay was 63 days (range $14-365)$ ).). Neonatal intensive care unit comorbidities are listed in Table 2. Seven children developed seizures, at a median age of 4 days (range 1 -25). Five children had tone abnormalities, 19 had feeding issues, none had hemiparesis.

\section{Thrombophilia evaluations}

Nine children were evaluated for prothrombotic disorders, and 7 had at least one: Factor $\mathrm{V}$ Leiden ( 2 of 9 evaluated were heterozygous, 0 of 9 were homozygous), MTHFR C677T ( 2 of 7 evaluated were heterozygous, 1 of 7 was homozygous), MTHFR A1298C (5 of 7 evaluated were heterozygous, 1 of 7 was homozygous), PAI-1 ( 1 of 2 tested was heterozygous, the other was homozygous.). None of the of 7 evaluated had the prothrombin 20210 mutation.

\section{Outcomes Table 3}

One child died in the neonatal intensive care unit (age of death 123 days), and all 22 (100\%) survivors had at least one disability; $18(82 \%)$ had at least moderate-severe disability. Median age at last follow-up was 51 months (range 14-202 months). All three children with cerebellar infarcts had cerebral palsy, all 9 children born before 31 weeks' gestational age had cerebral palsy, and 6 of the 7 surviving children with unilateral or bilateral grade 3 or 4 intraventricular hemorrhage had cerebral palsy (the $7^{\text {th }}$ had mild motor impairment), but we found it difficult to relate other disabilities to gestational age or location of cerebral injury.

\section{Discussion}

Premature children with arterial ischemic stroke during early infancy often have complicated gestational and perinatal courses, have additional neurological complications of prematurity in addition to their stroke, may have patterns of infarction that are rarely seen in term infants, have prolonged neonatal intensive care unit stays, and are frequently left with profound disability. Stroke appears to add to the impairments of prematurity, and prematurity might add to the impairment of very early stroke.

We chose to look at "very early stroke" rather than "perinatal" stroke in premature infants because diagnosing early stroke in the premature neonate is so challenging, and it is difficult to pinpoint exactly when these infarcts occur. Many premature neonates are too ill to be moved to the computed tomography scanner or magnetic resonance imaging scanner. Cranial ultrasonography can be performed in the neonatal intensive care unit at the bedside, may detect some cases of stroke in infants, but can miss acute, small, and posterior infarcts.[6,7] A group in the Netherlands did look at perinatal stroke in premature infants, but there were some interesting differences in their cohort. Benders and colleagues[8] used ultrasound to identify their 31 preterm infants with perinatal stroke. All of their patients were 27 weeks gestational age at birth or older; we had 6 children under 27 weeks gestational age at birth. There were no cerebellar infarcts in their cohort, probably because there were no extremely premature children; we had 3 children with cerebellar infarcts, all of whom were between 23 and 26 weeks gestational age at birth. Several other authors have noted cerebellar infarction in extremely premature neonates[9-11]. 
The limited data available on premature neonates with perinatal or "very early" infarction makes it difficult to examine risk factors associated with early infarction in this group. Benders and colleagues [8] found 8 pairs of twins (26\%) in their cohort of premature infants with perinatal arterial stroke, and found that 6 were part of monozygous twin pairs with associated twin-twin transfusion syndrome. They compared the children with stroke with matched controls, and found that twin-twin transfusion syndrome was an independent risk factor for perinatal stroke. We had 5 twins in our cohort (22\%), but only one case of twin-twin transfusion syndrome. Being a twin may itself be a risk factor for infarction, even in the absence of twintwin transfusion syndrome[12]. However, we did not have matched controls. Benders and colleagues[8] also found that fetal heart rate abnormality and hypoglycemia were risk factor for perinatal stroke in premature neonataes. We know that 6 children in our cohort had fetal distress, and 2 had hypoglycemia in the neonatal intensive care unit. We did have 6 children with meningitis, which has been described as a risk factor for stroke in term neonates[13,14]. Although prothrombotic risk factors have been described as a risk factor for stroke in term neonates[15], few of the infants in Benders' cohort[8] or ours received full prothrombotic workups, probably because they were unstable in the neonatal intensive care unit for prolonged periods and often had anemia from multiple blood draws required for ongoing care.

Previous work on perinatal arterial ischemic stroke in full-term neonates has described a male predominance[16]. We did not see a male predominance in this cohort. We do not know if this is due to chance, or if a male predominance does not occur in the premature stroke population. Perhaps if we were able to identify which infarcts did occur in the perinatal period, we would see a male predominance in premature children with truly perinatal strokes. We may be able to revisit this issue in the future.

The children in our paper had very long neonatal intensive care unit stays, with a median of 63 days (range 14-365). Neonatal intensive care unit stays vary across hospitals and among countries, making it difficult to compare these numbers to previously published norms [17, 18]. In our hospital, the median neonatal intensive care unit stay varies by year and gestational age, in an upside-down u-shaped curve. Length of stay are shorter for very premature infants, who may not survive, and less premature infants, with the longest stays for children around 25 weeks gestational age at birth. Between 2002 and 2004, the mean length of stay varied from 23-51 days for neonates $<=23$ weeks gestational age at birth, up to 90 days for children 25 weeks' gestational age at birth, to 32-28 days for children 29-33 weeks gestational age at birth (data from the Riley Hospital Neonatal Intensive Care Unit, personal communication). In general, the patients in our cohort appeared to have long stays for gestational age.

It is difficult to identify when these children clinically present with the first signs of stroke. Apnea and seizures are common presenting signs of common complications of prematurity (respiratory distress syndrome, intraventricular hemorrhage, periventricular leukomalacia), and are also common presenting signs of arterial ischemic stroke[19]. We describe when an infant has the first symptoms that may be indicative of a neurological problem, but it is not clear if these were the first signs of the child's stroke.

Premature children are at high risk for neurological disability even without arterial ischemic stroke, and the rate of disability increases dramatically as gestational age at birth drops. In children under 30 weeks' gestational age at birth, $10 \%$ have cerebral palsy and $17 \%$ have severe cognitive impairment[20]. In children under 26 weeks' gestational age at birth, $46 \%$ have moderate-severe disability[21]. The rates of impairment in our group were even higher; for the group as a whole, no child was free of disability, over $70 \%$ had cerebral palsy and over $40 \%$ had moderate-severe cognitive impairment and $>80 \%$ had at least one moderate-severe disability. 
Full-term neonates with periantal stroke who present during the newborn period are at high risk of disability. In our own cohort of term neonates with perinatal arterial ischemic stroke, among children who presented with stroke in the neonatal period, 54\% had cerebral palsy and $22 \%$ had moderate-severe cognitive impairment[1]. Approximately $67 \%$ of this cohort had childhood epilepsy, though almost a third of those children were able to be weaned off medication during the period that we followed them[22]. Again, the rates of cerebral palsy and moderate-severe cognitive impairment are higher in our premature cohort, but the differences are not statistically significant. Larger studies are needed to determine if premature infants with stroke have worse outcomes than term infants with stroke. So far, the incidence of epilepsy in premature infants with early stroke is not appreciably higher than in term children with perinatal stroke, but this may change as we obtain additional follow-up.

This study has several limitations. We may not have identified all premature neonates with infarction, either because we missed them with our search strategies, or because we missed them on cranial imaging. We may have missed small infarctions in children who had significant injury from large intraventricular hemorrhages or periventricular leukomalacia. We can revisit this issue in the future as imaging techniques in the premature neonate improve. Until we have more options for performing cerebral imaging on medically fragile premature neonates, it will be difficult to determine the true incidence of early and perinatal arterial infarction in this group.

Early arterial ischemic stroke in the premature infant appears to add to the neurological impact of prematurity alone. Further work is needed to understand the risk factors that contribute to infarction in this population, and we suggest that more of these children should receive prothrombotic evaluations. Advances in imaging the premature infant brain will aid in understanding when- and why- these infarcts occur.

\section{Acknowledgments}

Dr. Golomb is supported by grants from NIH NINDS (K23 NS 048024) and Clarian Values Fund (grant \#VFR-171). Dr. Williams is supported by NINDS R01 NS 39571 and Veterans' Administration Stroke QUERI STR 03-168.

\section{References}

1. Golomb MR, Saha C, Garg BP, Azzouz F, Williams LS. Association of cerebral palsy with other disabilities in children with perinatal arterial ischemic stroke. Pediatr Neurol 2007;37:245-249. [PubMed: 17903667]

2. Golomb MR, deVeber GA, MacGregor DL, Domi T, Whyte H, Stephens D, Dick PT. Independent walking after neonatal arterial ischemic stroke and sinovenous thrombosis. J Child Neurol 2003;18:530-536. [PubMed: 13677578]

3. deVeber GA, MacGregor D, Curtis R, Mayank S. Neurologic outcome in survivors of childhood arterial ischemic stroke and sinovenous thrombosis. Journal of Child Neurology 2000;15:316-324. [PubMed: 10830198]

4. Golomb MR, Garg BP, Saha C, Williams LS. Accuracy and yield of ICD-9 codes for identifying children with ischemic stroke. Neurology 2006;67:2053-2055. [PubMed: 17159120]

5. Golomb MR, Garg BP, Saha C, Azzouz F, Williams LS. The association of cerebral palsy with other disability in children with perinatal arterial ischemic stroke. Pediatr Neurol 2007;37:245-249. [PubMed: 17903667]

6. Golomb MR, Dick PT, MacGregor DL, Armstrong DC, deVeber GA. Cranial ultrasonography has a low sensitivity for detecting arterial ischemic stroke in term neonates. J Child Neurol 2003;18:98-103. [PubMed: 12693775]

7. Cowan F, Mercuri E, Groenendaal F, Bassi L, Ricci D, Rutherford M, de Vries L. Does cranial ultrasound imaging identify arterial cerebral infarction in term neonates? Arch Dis Child Fetal Neonatal Ed 2005;90:F252-F256. [PubMed: 15846018] 
8. Benders MJ, Groenendaal F, Uiterwaal CS, Nikkels PG, Bruinse HW, Nievelstein RA, de Vries LS. Maternal and infant characteristics associated with perinatal arterial stroke in the preterm infant. Stroke 2007;38:1759-1765. [PubMed: 17495219]

9. Mercuri E, He J, Curati WL, Dubowitz LM, Cowan FM, Bydder GM. Cerebellar infarction and atrophy in infants and children with a history of premature birth. Pediatr Radiol 1997;27:139-143. [PubMed: 9028846]

10. Rollins NK, Wen TS, Dominguez R. Crossed cerebellar atrophy in children: a neurologic sequela of extreme prematurity. Pediatr Radiol 1995;25:S20-S25. [PubMed: 8577528]

11. Johnsen SD, Bodensteiner JB, Lotze TE. Frequency and nature of cerebellar injury in the extremely premature survivor with cerebral palsy. J Child Neurol 2005;20:60-64. [PubMed: 15791925]

12. Golomb MR, Williams LS, Garg BP. Perinatal stroke in twins without co-twin demise. Pediatr Neurol 2006;35:75-77. [PubMed: 16814092]

13. Ment LR, Ehrenkranz RA, Duncan CC. Bacterial meningitis as an etiology of perinatal cerebral infarction. Pediatric Neurology 1986;2:276-279. [PubMed: 3508700]

14. Fitzgerald KC, Golomb MR. Neonatal arterial ischemic stroke and sinovenous thrombosis associated with meningitis. J Child Neurol 2007;22:818-822. [PubMed: 17715272]

15. Golomb MR. The contribution of prothrombotic disorders to peri- and neonatal ischemic stroke. Semin Thromb Hemost 2003;29:415-424. [PubMed: 14517753]

16. Golomb MR, Dick PT, MacGregor DL, Curtis R, Sofronas M, deVeber GA. Neonatal arterial ischemic stroke and cerebral sinovenous thrombosis are more commonly diagnosed in boys. J Child Neurol 2004;19:493-497. [PubMed: 15526952]

17. McCormick MC, Escobar GJ, Zheng Z, Richardson DK. Place of birth and variations in management of late preterm ("near-term") infants. Semin Perinatol 2006;30:44-47. [PubMed: 16549213]

18. Profit J, Zupancic JA, McCormick MC, Richardson DK, Escobar GJ, Tucker J, Tarnow-Mordi W, Parry G. Moderately premature infants at Kaiser Permanente Medical Care Program in California are discharged home earlier than their peers in Massachusetts and the United Kingdom. Arch Dis Child Fetal Neonatal Ed 2006;91:F245-F250. [PubMed: 16449257]

19. Volpe, JJ. Neurology of the Newborn. Philadelphia: W.B. Saunders Company; 2001.

20. Woodward LJ, Anderson PJ, Austin NC, Howard K, Inder TE. Neonatal MRI to predict neurodevelopmental outcomes in preterm infants. N Engl J Med 2006;355:685-694. [PubMed: 16914704]

21. Marlow N, Wolke D, Bracewell MA, Samara M. Neurologic and developmental disability at six years of age after extremely preterm birth. N Engl J Med 2005;352:9-19. [PubMed: 15635108]

22. Golomb MR, Garg BP, Carvalho KS, Johnson CS, Williams LS. Perinatal stroke and the risk of developing childhood epilepsy. J Pediatr 2007;151:409-413. [PubMed: 17889079]413 e401-402 

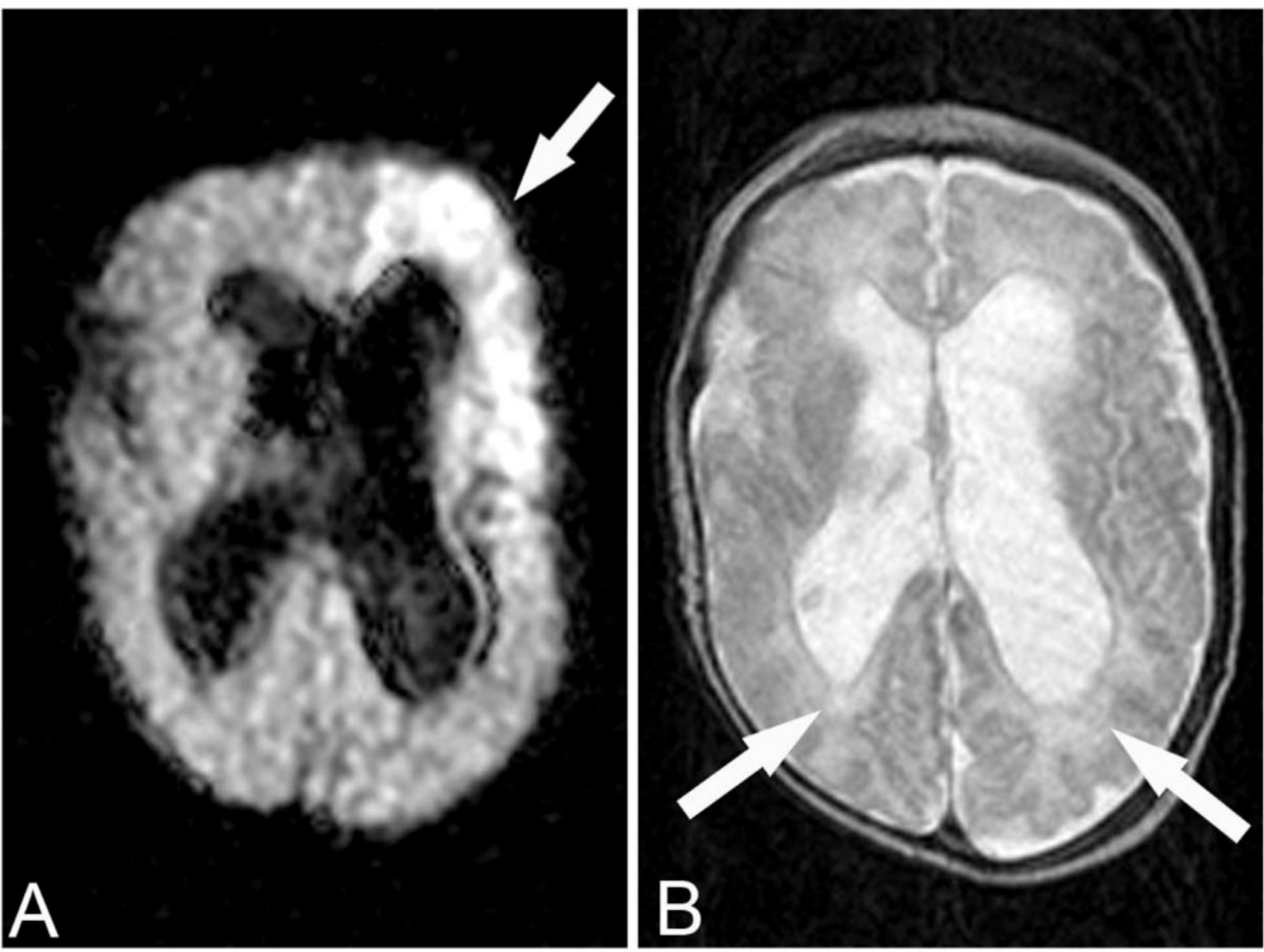

Figure 1.

This child was born at 23 weeks gestational age. This MRI was performed at approximately 40 weeks gestational age. A. Diffusion-weighted imaging (TR 7,000 TE 140.5) demonstrated acute infarction in the left middle cerebral artery territory (arrow). B. Fast spin echo T2weighted imaging (TR 3,500 TE 100) demonstrates severe perinventricular leukomalacia (arrows). She has significant cerebral palsy and cognitive impairment. 

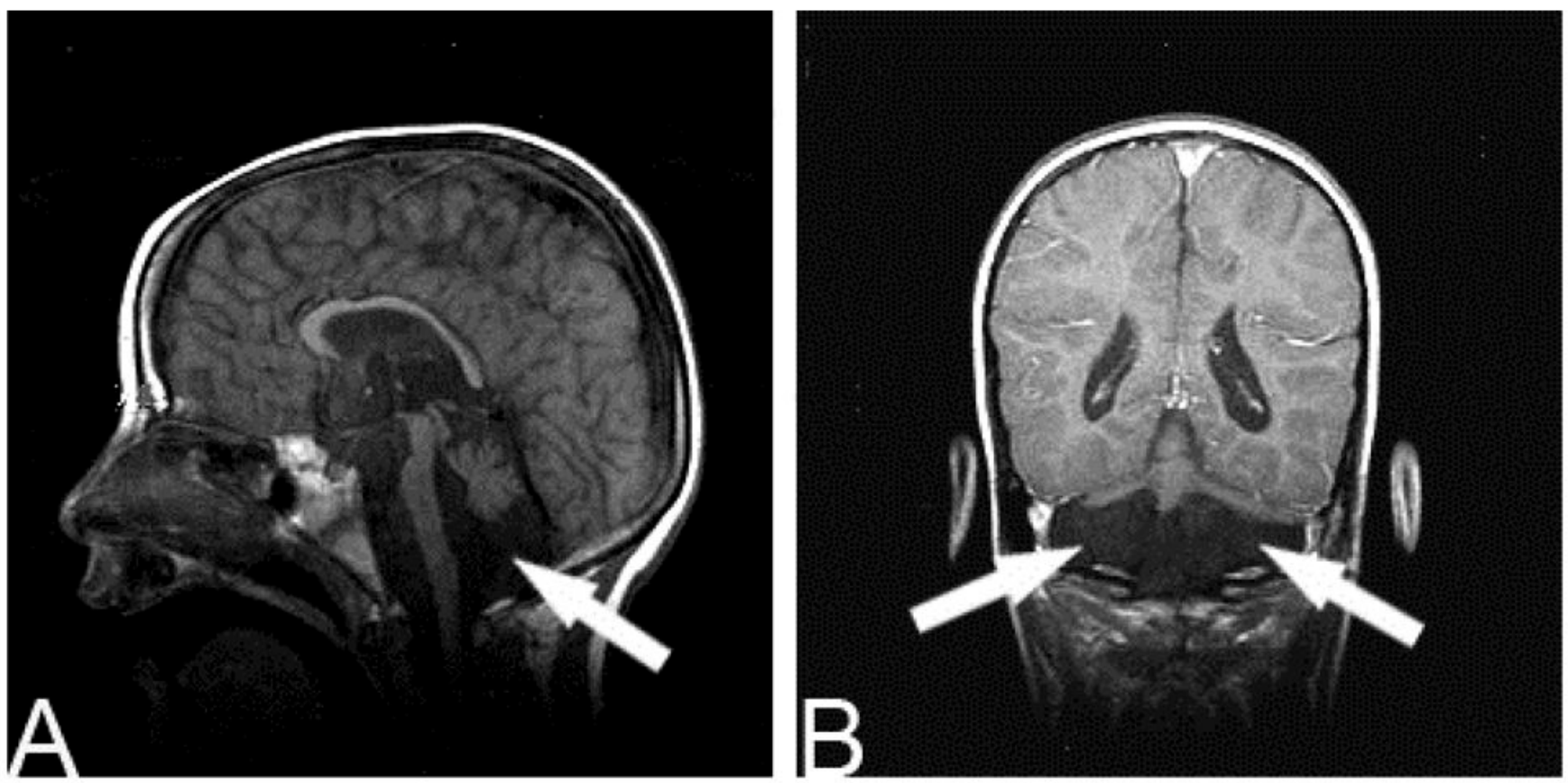

Figure 2.

This child was born at 26 weeks gestational age and was found to have bilateral cerebellar infarcts (arrows; A sagittal T1 view, TR 450 TE 14; B. coronal T1 + contrast view, TR 516.664 $T E 20)$. He has profound motor and cognitive disability. 
Table 1

Gestational age and weight at birth $\mathrm{N}=23$

\begin{tabular}{lll}
\hline Gestational age at birth & $\#(\%)$ & birth weight range for that age (gm) \\
\hline 23 & $2(9 \%)$ & $645-645$ \\
24 & $1(4 \%)$ & 632 \\
25 & $1(4 \%)$ & 920 \\
26 & $2(9 \%)$ & $690-765$ \\
27 & $3(13 \%)$ & $755-1185$ \\
28 & $1(4 \%)$ & 535 \\
29 & $0(0 \%)$ & - \\
30 & $0(0 \%)$ & $1317-1511$ \\
31 & $4(17 \%)$ & $1304-2410$ \\
32 & $2(9 \%)$ & $1875-1956$ \\
33 & $2(9 \%)$ & 1980 \\
34 & $1(4 \%)$ & $2245-2786$ \\
35 & $4(17 \%)$ & \\
\hline
\end{tabular}


Table 2

Neonatal Intensive Care Unit Comorbidities

\begin{tabular}{lll}
\hline Cormorbidity & $\mathbf{N}=\mathbf{2 3}$ & $(\mathbf{\%})$ \\
\hline Respiratory complications & $\#$ & $(83 \%)$ \\
Intubation & 19 & \\
Pneumonia & 19 & $(4 \%)$ \\
Bronchopulmonary dysplasia & 4 & $(17 \%)$ \\
Extracorporeal Membrane Oxygenation & 8 & \\
Cardiac Abnormality & 1 & \\
Complex congenital heart disease & 4 & $(35 \%)$ \\
Atrial septal defect & 0 & \\
Patent ductus arteriosus & 1 & \\
Patent ductus arteriosus + atrial septal defect & 2 & $(83 \%)$ \\
Pressor Support & 1 & $(4 \%)$ \\
Dopamine & 8 & $(43 \%)$ \\
Dopamine + Dobutamine & 4 & $(9 \%)$ \\
Epinephrine & 3 & $(26 \%)$ \\
Chest compressions + epinephrine & 1 & $(52 \%)$ \\
Feeding issues & 2 & $(4 \%)$ \\
Necrotizing enterocolitis & 19 & \\
Sepsis & 1 & \\
Suspected sepsis, negative cultures & 10 & \\
Meningitis & 2 & \\
Anemia & 6 & \\
Hypoglycemia & 12 & \\
Thrombus to left foot resulting in amputation & 2 & \\
\hline
\end{tabular}




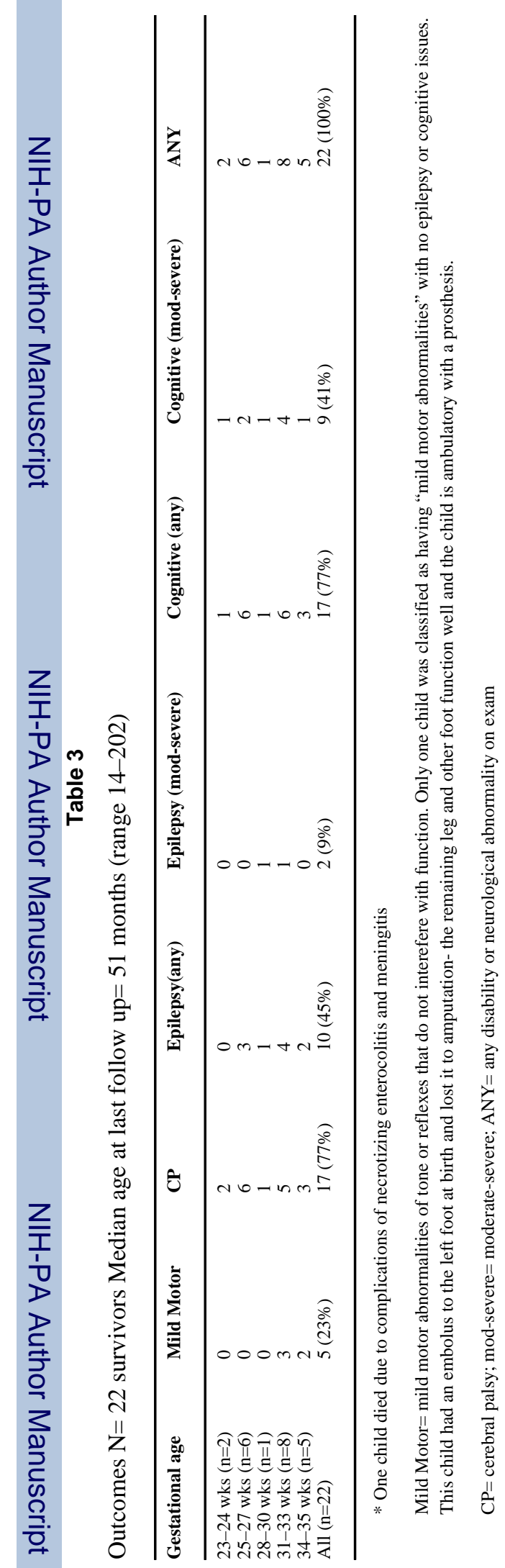

Pediatr Neurol. Author manuscript; available in PMC 2009 October 30. 